\title{
Implementing green chemistry. An environmental policy for sustainability*
}

\author{
Joe Thornton \\ Columbia Earth Institute, Columbia University \#2430, New York, NY 10027, USA
}

\begin{abstract}
A diverse cocktail of synthetic chemicals is now globally distributed in the environment, food web, and human tissues, posing significant health risks to humans and wildlife. The current approach to environmental regulation is inherently inadequate to address the scope and complexity of this problem. A fundamental shift in paradigm is required to protect human health and ecological systems from chemically induced damage. The concept of green chemistry represents a critical part of this shift, but it is not sufficient per se. Only if green chemistry is conceived as part of a new policy based upon precautionary, democratically guided implementation of sustainable production technologies will it contribute significantly to establishing an ecologically compatible production base.
\end{abstract}

\section{GLOBAL CONTAMINATION AND HEALTH HAZARDS}

With explosive global growth since World War II, the synthetic chemical industry now produces billions of tons per year of up to 70000 commercial substances that are used for diverse technical and economic purposes [1]. Many of these chemicals and the by-products produced during their life cycle are stable and/or oil-soluble, causing them to persist in the environment and accumulate in food webs [2]. Longlived pollutants are distributed long distances on currents of wind and water and have accumulated on a truly global basis [3]. Although research and policy have focused primarily on a handful of substances-PCBs, dioxin, and about a dozen pesticides - global contamination cannot be reduced to a few "bad actors". In the Great Lakes, for example, 362 synthetic chemicals have been "unequivocally identified" in the water, sediments, and food chain; the list includes the most infamous pollutants, but it also contains a full spectrum of less-familiar substances, from simple solvents to complex chlorinated polyaromatics and by-products [4]. At least 700 xenobiotic pollutants are present in the adipose tissues of the general population of the United States [5].

A growing body of evidence indicates that exposure to some of these pollutants, even in very low doses, can cause severe health impacts. Laboratory studies indicate that a large number of synthetic substances can disrupt the endocrine, reproductive, immune, and nervous systems, as well as disrupting development and contributing to cancer and other diseases. Sensitivity is particularly high when exposure occurs in utero or early in development. For several pollutants, health impacts have been observed at body burdens in the same range as are found in the general human population [6,7]. Bioaccumulated substances in such large ecosystems as the Great Lakes and the Baltic and Wadden Seas are high enough to have caused severe and widespread health impacts in wildlife populations [8-10]. Humans exposed to higher than background doses have suffered similar health effects, and numerous studies suggest that the low-level exposures to which the general public is subject may also increase health risks of several types (reviewed in ref. 2).

\footnotetext{
*Lecture presented at the IUPAC CHEMRAWN XIV Conference on Green Chemistry: Toward Environmentally Benign Processes and Products, Boulder, Colorado, USA, 9-13 June 2001. Other presentations are published in this issue, pp. 1229-1330.
} 
These considerations indicate that universal chemical exposure poses significant risks to the health of humans and wildlife on a universal scale. Chemical pollution has become a problem of global reach and long-term severity, like global warming, ozone depletion, and loss of biodiversity. It requires solutions of similar ambition and scope.

\section{GREEN CHEMISTRY: NECESSARY BUT NOT SUFFICIENT}

The growing field of green chemistry-chemical engineering with the specific goal of designing environmentally sustainable products and processes-reflects an understanding that the pollution control and disposal regime that has dominated environmental policy since the 1970s has not been successful at preventing large-scale chemical pollution. The predominant effect of control and disposal devices is to shift chemicals from one environmental medium, place, or chemical form to another; eventually, captured pollutants make their way into the ecosystem in one place or another, in one form or another. Even incinerators, which have become the technological centerpiece of the control and disposal regime, are now leading sources of many metallic pollutants and the largest identified sources of dioxins, which are inevitably formed as products of incomplete combustion when organochlorine wastes are burned $[11,12]$. Moreover, pollution control strategies do nothing to reduce environmental releases of substances that are deliberately dissipated into the environment or the economy, such as pesticides, paint strippers, and plastics, and therefore cannot be subject to on-site pollution control.

Reducing inputs of toxic substances by redesigning products and processes prevents the production of hazardous chemicals and wastes at the source and thereby eliminates emissions and exposures altogether. Green chemistry is therefore an essential part of the solution to global chemical contamination. In itself, however, development of green chemistry is not an adequate remedy, because safer technologies will be implemented on a large scale only if society adopts an environmental policy framework that departs radically from the current one. In the current system, safer alternatives exist for many sources of hazardous chemicals, but in many cases they are not used widely. For example, effective chlorine-free pulp bleaching methods, which produce none of the persistent organochlorine pollutants associated with chlorine-based bleaches, are used in dozens of state-of-the-art pulp mills, primarily in Europe, but only a few facilities in the United States have adopted these methods. Environmentally superior alternatives to perchloroethylene in clothes cleaning, substitutes for PVC plastic in construction materials, and methods to reduce the use of synthetic pesticides in agriculture have been adopted to only a limited extent.

\section{ENVIRONMENTAL POLICY BARRIERS TO GREEN CHEMISTRY}

The critical question is why more sustainable technologies have languished. The proximate causes are lack of knowledge, institutional inertia, capital costs of conversion, and, in some cases, increases in operating costs. The ultimate reason why most firms have not invested in safer products and processes is that current policies have put little or no pressure upon them to do so. Indeed, the current framework for environmental regulation perpetuates the use of polluting technologies and is, therefore, in significant part responsible for continuing global contamination.

In the current regime, society attempts to manage pollution by permitting chemical production, use, and release, as long as discharges of individually regulated substances from individual facilities do not exceed some quantitative standard of acceptable contamination. The central artifact of this approach is the pollution permit, which sets maximum legal release rates of individual toxic chemicals from individual facilities; a similar approach is used to establish occupational exposure limits and maximum contaminant levels in drinking water and on foodstuffs. These levels are usually set using risk assessment, based on extrapolations of toxicological studies and numerous assumptions about pollutant fate, transport, and exposure to back-calculate a discharge limit that is predicting to be safe for a "most exposed 
individual" in the vicinity of the facility. Industries typically comply with permitted limits by installing pollution control devices or improving housekeeping practices.

The effect of this regulatory regime is to protect firms from pressure to adopt safer technologies, for several reasons. First, permitting discharges within "acceptable" limits perpetuates reliance on pollution control, because it is virtually always possible to meet permit limits by improving or installing additional control devices; it is only necessary to change materials and production processes themselves when the acceptable limit is zero. And as long as discharges are within the acceptable limit, the existence of alternative technologies that do not produce potentially hazardous chemicals is never examined. But permitting "acceptable" discharges can never prevent global contamination for substances that persist or bioaccumulate, because these chemicals, even if discharged in very small amounts, build up gradually in the environment and eventually reach unacceptable levels. The only discharge rate of such substances that is compatible with ecological processes is zero.

Second, the current framework's focus on individual facilities and the local environments around them fails to take account of the global, cumulative nature of chemical pollution and thus permits the continuing use of hazardous technologies. When a facility is granted a permit to discharge pollutants into the air or water, the risk assessments on which these decisions are based consider only local contamination and immediate exposures through one or a few pathways. Any pollutants distributed beyond the local environment and the most exposed individual disappear entirely from the risk assessment process. Moreover, no attention is paid to the fact that there may be hundreds of other facilities licensed to release the same substance, each of which contributes to a global burden that is never considered. Preventing severe local contamination from each of a myriad of individual facilities, operating simultaneously, year after year, does not prevent the slow accumulation of a global pollution burden.

Third, the current regime's focus on individual substances does not take account of the full life cycle of chemical products; it therefore systematically underestimates the environmental hazards of those substances and the benefits of replacing them with cleaner substitutes. Scores to hundreds of byproducts have been identified in the manufacture, use, and disposal of halogenated synthetic chemicals [2], for example, but these pollutants are not considered in the assessment and licensing of the product itself. When, for example, a chemical manufacturer is permitted to produce and discharge vinyl chloride, the formation of dioxins, PCBs, and other highly persistent substances as accidental by-products during the synthesis of vinyl chloride or the incineration of polyvinyl chloride plastic $[13,14]$ are not part of the assessment of vinyl chloride's risks. Indeed, the continuing discharge of many tons of PCBs into the environment each year as by-products of a range of chlorine-based industrial processes $[2,15]$, decades after the deliberate production of PCBs was phased out, illustrates the limits of policies focused on the discharge of individual pollutants rather than entire life cycles. Moreover, the vast majority of by-products in most processes have not yet been specifically identified, due to the limitations of analytic technologies $[16,17]$. The risk of unidentified substances cannot be specifically assessed, so the chemical-specific licensing process ignores these by-products altogether, although they may pose substantial hazards.

Finally, the current framework's focus on individual chemicals also systematically underestimates the hazards of toxic technologies. The information necessary to evaluate with confidence the health hazards of the thousands of synthetic chemicals in commerce simply do not exist. Even among the subset of high-volume chemicals that are already subject to specific regulations-those expected to have been the most thoroughly studied-70\% lack even minimal chronic toxicity data [18]. The U.S. National Toxicology Program conducts assessments for 10 to 20 substances per year [19], but 200-300 new chemicals are introduced annually, so our knowledge base is falling further behind all the time [1]. Even for those chemicals for which toxicological data are available, virtually all testing has been done on the substance in isolation; toxicological synergy is common, however, and levels predicted to be safe based on single-chemical assessments often turn out to cause health effects in the context of exposure to mixtures [20]. The general lack of environmentally relevant data presents a real problem in a regime that presumes synthetic chemicals harmless until they are demonstrated hazardous. In a risk assessment, a 
chemical for which there are no toxicological data is assigned a risk of zero, and a substance with a zero risk is subject to no restrictions. The result is that the vast majority of chemicals receive laissez-faire treatment simply because they have been studied superficially, if at all. Hazardous technologies are therefore licensed and the existence of cleaner alternatives ignored based upon predictions that the public's exposure burden is safe, despite the fact that these predictions often have little or no empirical basis.

These dynamics combine to make the current policy framework unsuitable for addressing the global, cumulative hazards posed by large-scale use of hazardous chemicals. The narrow focus on quantifying and managing local, short-term risks excludes consideration of the long-term impacts of the global pollutant burden that results from the widespread use of dangerous chemicals. The result is that hazardous technologies are licensed and perpetuated, and questions that ought to be at the center of the policy process are marginalized: how much risk is acceptable, what standard of proof should be used in environmental decision-making, and, most importantly, how can society fulfill its economic needs in a way that does not risk compromising human health and the environment?

\section{A POLICY FRAMEWORK TO ENCOURAGE SUSTAINABLE PRODUCTION}

In the absence of a policy framework that systematically moves society toward more sustainable production methods, green chemistry will remain a niche discipline, the industrial equivalent of organic food and environmentally friendly building materials. In turn, global contamination will continue. An alternative framework - the Ecological Paradigm, which I have described elsewhere in detail [2]should begin by recognizing that our ability to predict, manage, and diagnose the global impacts of complex chemical discharges is very limited, particularly at the fine scale required to control individual substances. We should therefore not make risky bets based on those predictions or wait to protect health and the environment until we have conclusive proof of harm. Instead, the Precautionary Principle instructs us to avoid practices that have the potential to cause damage that is severe, widespread, and/or irreversible, even in the absence of proof [21].

The precautionary principle does not specify what kinds of action to take, so the Ecological Paradigm relies on four other principles, which are formulated specifically to address the limits of the current approach. First is the principle of Zero Discharge: to prevent the long-term accumulation and global dissemination of pollutants, substances that are persistent or bioaccumulative are considered incompatible with ecological processes, and releases in any quantity are treated as a potential hazard. The goal of policy becomes not the licensing of "acceptable" discharges but eliminating releases of such substances altogether [22].

To accomplish this goal, the Ecological Paradigm takes a new technological approach with Clean Production [23]. In contrast to the after-the-fact strategy of pollution control and disposal, Clean Production emphasizes front-end solutions, particularly the redesign of products and processes to eliminate the use and generation of toxic chemicals, before they need to be managed. The principle of Clean Production says simply that we should always use the cleanest available technology to fulfill society's needs. Assessment of alternatives rather than approval of pollution becomes the centerpiece of environmental management [24].

To address the overwhelming lack of information that undermines the chemical-by-chemical approach, the Ecological Paradigm shifts the burden of proof, an idea called Reverse Onus. In the current system, a lack of data is misconstrued as evidence of safety, and all the untested and unidentified chemicals are assumed to be safe. In the Ecological Paradigm, the burden of proof shifts to those who want to produce or use a synthetic chemical, who must demonstrate in advance that their actions are not likely to pose a significant hazard and/or that there is no safer alternative available. In this way, a lack of conclusive data on hazards is not allowed to stand in the way of implementing less-polluting alternatives if they exist. 
Finally, to cope with the impossibility of micromanaging thousands of substances produced by thousands of facilities, the scale of preventative measures in the Ecological Paradigm shifts from micromanagement of individual sources and substances to classes of chemicals and the processes that produce them. Priority in environmental policy is given to those classes whose members tend to have the most hazardous qualities. Organohalogens and toxic metals, which tend to be persistent and/or bioaccumulative for well-understood chemical reasons [2], are obvious choices for immediate action.

The Ecological Paradigm implies significant technological change, so it requires a carefully planned process called chemical sunsetting [25]. Sunsetting begins by reversing the onus for classes of chemicals and processes for which a prima facie case can be established that their members tend to be hazardous. Members of these classes are treated as candidates for replacement with safer alternatives; specific exceptions are made for uses that can be demonstrated to a reasonable degree of certainty to be nonhazardous or to fulfill an important societal need for which no alternatives are available. The goal of environmental policy becomes avoiding these substances, and progress is made toward that goal by converting their sources to cleaner materials and processes. Persistent synthetic chemicals are used in a wide variety of industrial processes, so the complete conversion to safer technologies will take place over decades. Chemical sunsets require priorities and timelines to be established, exceptions to be granted, and the safest and most effective substitutes to be chosen; these decisions should be made democratically and should be specifically tailored to each nation or region.

\section{INNOVATION IN SUSTAINABLE PRODUCTION PROCESSES}

The result of the Ecological Paradigm would be progressive reductions in the use of synthetic chemicals, except those designed specifically or shown to be compatible with ecological and physiological processes. This approach provides a framework to drive the gradual transformation of current industrial technology, with its countless individual hazards, into a sustainable infrastructure for economic production. Green Chemistry, of course, would play a key role in technological research and development. Although safer alternatives exist already for many applications of hazardous chemicals, there are certain uses for which effective substitutes are not yet available [2,26]. Further, most existing alternatives are not perfect, so there is room for environmental and economic improvement. The Ecological Paradigm would provide a framework of mandates and incentives for continual gains in sustainability via technological development.

A broad policy for sustainable production is critical. Risk-based regulations dominate most environmental policy, but facets of the Ecological Paradigm have been implemented ad hoc, and these actions have been far more successful at reducing pollution than rules based on pollution control and risk-based limits. For example, restrictions on production and use of DDT and other pesticides, PCBs, CFCs, and lead have led to drastic reductions in releases, and environmental levels and human exposures have gradually followed [27]. But even these accomplishments are only partial successes, because many restricted substances have been replaced by other hazardous chemicals, such as HCFCs and HFCs for CFC refrigerants, and 2,4-D and triazine herbicides for 2,4,5-T. These examples show that chemicals must be controlled in the context of a broad mandate to deploy environmentally sound production methods, so that society does not replace one bad product with another, but moves continually towards the most sustainable technologies. With such a mandate, many of the errors of the Chemical Age could be gradually repaired.

\section{REFERENCES}

1. Organization for Economic Cooperation and Development. OECD Environmental Outlook for the Chemicals Industry, OECD, Paris (2000).

2. J. Thornton. Pandora's Poison: Chlorine, Health, and a New Environmental Strategy. MIT Press, Cambridge (2000).

(C) 2001 IUPAC, Pure and Applied Chemistry 73, 1231-1236 
3. S. L. Simonich and R. A. Hites. Science 269, 1851-1854 (1995).

4. Great Lakes Water Quality Board. 1987 Report on the Great Lakes Water Quality. International Joint Commission, Windsor, Ontario (1987).

5. J. Onstot, J. R. Ayling, J. Stanley. Characterization of HRGC/MS Unidentified Peaks from the Analysis of Human Adipose Tissue, Volume I: Technical Approach. U.S. Environmental Protection Agency, Washington DC (1987).

6. M. J. DeVito, L. S. Birnbaum, W. H. Farland, T. Gasiewicz. Environ. Health Perspect. 103, 820-831 (1995).

7. E. M. Ulrich, A. Caperell-Grant, S. H. Jung, R. A. Hites, R. M. Bigsby. Environ. Health Perspect. 108, 973-977 (2000).

8. J. P. Giesy, J. P. Ludwig, D. J. Tillitt. Environ. Sci. Technol. 28, 128a-134a (1994).

9. R. L. de Swart, P. S. Ross, J. G. Vos, A. D. Osterhaus. Environ. Health Perspect. 104 (supp 4), 823-828 (1996).

10. A. Brouwer, P. Reinjders, J. H. Koeman. Aquat. Toxicol. 15, 99-106 (1989).

11. U.S. Environmental Protection Agency. The Inventory of Sources of Dioxin in the United States. Washington, DC (1998).

12. U.S. Agency for Toxic Substances and Disease Registry. Public Health Statement: Lead. U.S. Centers for Disease Control, Atlanta (1990).

13. United Kingdom Environment Agency. Regulation of dioxin releases from the Runcorn operations of ICI and EVC. Warrington, UK (1997).

14. Dow Chemical. Waste Analysis Sheet: Heavy Ends from the Distillation of Ethylene Dichloride in Ethylene Dichloride Production, Plaquemine, LA, February 21, 1990.

15. R. E. Alcock, P. A. Behnisch, K. C. Jones, H. Hagenmaier. Chemosphere 37, 1457-1472 (1998).

16. L. R. Suntio, W. Y. Shiu, D. Mackay. Chemosphere 17, 1249-1290 (1988).

17. U.S. Environmental Protection Agency. Background Document for the Development of PIC Regulations from Hazardous Waste Incinerators. Washington DC (1989).

18. Environmental Defense Fund. Toxic Ignorance: The Continuing Absence of Basic Health Testing for Top-Selling Chemicals in the United States, New York (1997).

19. G. W. Lucier and A. Schecter. Environ. Health Perspect. 106, 623-627 (1998).

20. R. S. H. Yang (Ed.). Toxicology of Chemical Mixtures. Academic Press, New York (1994).

21. C. Raffensperger and J. Tickner (Eds.). Protecting Public Health and the Environment: Implementing the Precautionary Principle. Island Press, Washington DC (1999).

22. International Joint Commission. Fifth Biennial Report on Great Lakes Water Quality, Windsor, Ontario (1990).

23. T. Jackson (Ed.). Clean Production Strategies: Developing Preventive Environmental Management in the Industrial Economy, Lewis, Boca Raton (1993).

24. M. O’Brien. Making Better Environmental Decisions: An Alternative to Risk Assessment, MIT Press, Cambridge (2000).

25. International Joint Commission. Sixth Biennial Report on Great Lakes Water Quality, Windsor, Ontario (1992).

26. Chem-Info Services. A Technical and Socio-Economic Comparison of Options to Products Derived from the Chlor-alkali Industry, Environment Canada, Burlington, Ontario (1997).

27. B. Commoner. Making Peace With the Planet, Pantheon, New York (1990). 